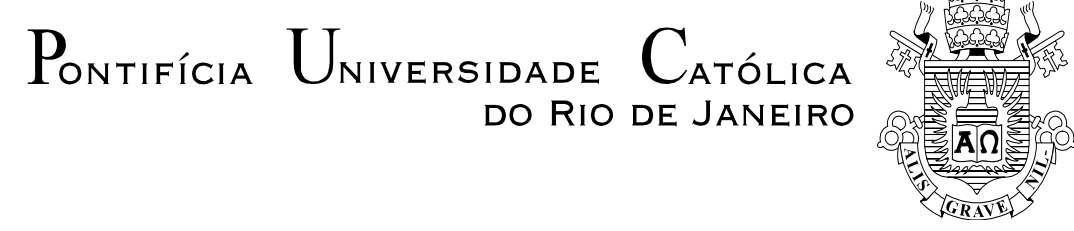

Keila Mara Cassiano

\title{
Análise de Séries Temporais Usando Análise Espectral Singular (SSA) e Clusterização de Suas Componentes \\ Baseada em Densidade
}

\section{Tese de Doutorado}

Tese apresentada ao Programa de Pós-graduação em Engenharia Elétrica do Departamento de Engenharia Elétrica da PUC-Rio como requisito parcial para obtenção do título de Doutor em Engenharia Elétrica.

Orientador: Prof. Reinaldo Castro Souza 


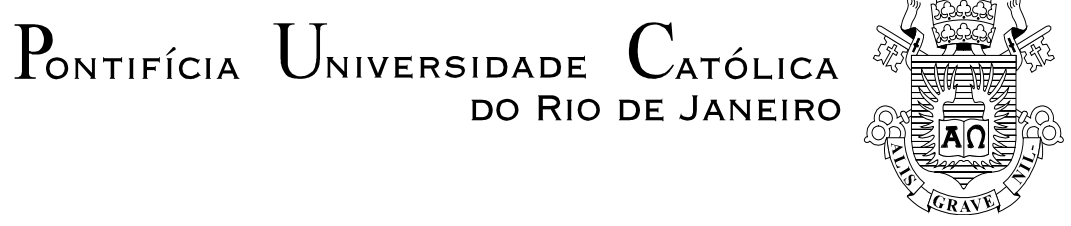

Keila Mara Cassiano

Análise de Séries Temporais Usando Análise Espectral Singular (SSA) e Clusterização de Suas Componentes Baseada em Densidade

\begin{abstract}
Tese apresentada como requisito parcial para obtenção grau de Doutor pelo Programa de Pós-Graduação em Engenharia Elétrica do Departamento de Engenharia Elétrica do Centro Técnico Científico da PUC-Rio. Aprovada pela Comissão Examinadora abaixo assinada.
\end{abstract}

Prof. Reinaldo Castro Souza

Orientador

Departamento de Engenharia Elétrica - PUC-Rio

Prof. ${ }^{a}$ Ana Maria Lima de Farias

UFF

Prof. Basílio de Bragança Pereira UFRJ

Prof. José Francisco Moreira Pessanha UERJ

Prof. ${ }^{a}$ Karla Tereza Figueiredo Leite Departamento de Engenharia Elétrica - PUC - Rio

Prof. José Eugenio Leal

Coordenador Setorial do Centro Técnico Científico - PUC-Rio

Rio de Janeiro, 12 de setembro de 2014. 
Todos os direitos reservados. É proibida a reprodução total ou parcial do trabalho sem autorização da Universidade, do autor e do orientador.

\section{Keila Mara Cassiano}

É Bacharel em Matemática pela Universidade Federal de Goiás, possui Mestrado em Estatística pela Universidade Federal de Pernambuco. Desenvolveu junto com o seu orientador uma grande quantidade de trabalhos e artigos científicos apresentados em congressos nacionais e internacionais. Também publicou artigos em revistas internacionais durante o Doutorado em Engenharia Elétrica da PUC-Rio.

Ficha Catalográfica

Cassiano, Keila Mara
Análise de Séries Temporais Usando Análise
Espectral Singular (SSA) e Clusterização de Suas
Componentes Baseada em Densidade/ Keila Mara
Cassiano; orientador: Reinaldo Castro Souza. - 2014.
172 f. : il. (color.) ; $30 \mathrm{~cm}$
Tese (doutorado) - Pontifícia Universidade
do Rio de Janeiro, Departamento de
Inclui bibliografia
1. Engenharia Elétrica - Teses. 2. Singular
Engenharia Elétrica, 2014.
Analysis. 3. Clusterização Baseada em
Spectrum
Densidade (DBSCAN) 4. Previsão de Séries Temporais.
5. Previsão SSA. 6. Modelos ARIMA. 7. Energia Eólica.
I. Souza, Reinaldo Castro. II. Pontifícia Universidade
Católica do Rio de Janeiro. Departamento de
Engenharia Elétrica. Ill. Título.

CDD: 621.3 
Para meus tesouros

Moisés Menezes,

Pedro Arthur

e Matheus Henrique,

com todo meu amor. 


\section{Agradecimentos}

Agradeço a Deus pela vida e por Seu cuidado, por me fortalecer, proteger, capacitar e iluminar a cada dia, permitindo que eu chegasse até aqui.

Ao meu esposo Moisés, pelo seu nobre amor, cuidado e parceria. Pela paciência e compreensão nas minhas ausências, por ser a calma nos momentos de preocupação, por ser sempre minha fortaleza, acreditar quando eu não acreditei, e me guiar nas decisões quando eu estava desorientada.

Aos meus amados filhos Pedro Arthur e Matheus Henrique por tantas vezes serem abdicados da minha atenção, carinho e dedicação e mesmo assim iluminarem meus dias com doces momentos que equilibram as tensões dos períodos de estudos e me nutrem de prazer e felicidade plenos.

Ao professor Reinaldo Souza, pela sua orientação segura, por seu conhecimento e disponibilidade, pelo apoio e acolhimento nos momentos críticos que tive neste doutorado. Pela calma, confiança e otimismo que ele sempre me transferiu e, principalmente pela compreensão e paciência diante das minhas falhas.

Ao Professor José Francisco Pessanha, por todas as colaborações, orientações e ideias enriquecedoras, pela parceria em diversos trabalhos, disponibilidade exemplar, amizade, apoio e incentivo constantes.

Aos meus pais, Iva Cassiano e Antônio Cassiano, por sempre terem feito o melhor pra promoverem meu desenvolvimento e crescimento. Por sempre acreditarem na minha capacidade, me incentivando e apoiando em todos os momentos, especialmente nestes últimos anos problemáticos.

Às minhas doces avós Adelaide e Manoelina que estão sempre intercedendo por mim e enchendo minha vida de candura e amor.

Aos meus familiares que sempre me auxiliaram diretamente, principalmente às minha queridas Dadá e Dedé, Greice Carla e Kênia Cristina, Kátia Kelvis e Carlos Lozano, pelo apoio e ajuda que me deram quando eu precisei. 
À minha amiga-filha Danielle Freitas, pela amizade, carinho e compreensão. Aos meus médicos Dr. Rodrigo e Dra. Olga, que foram muito mais que médicos, realmente amigos, me ajudando a superar e vencer os desafios dos momentos de enfermidade com profissionalismo e dedicação incomum.

Aos professores do Departamento de Engenharia Elétrica da PUC-Rio pela competência e pelo conhecimento transferido à minha formação.

Ao Professor Alexandre Street por sua orientação inicial, pois sem seu apoio e acolhimento eu não teria ingressado neste curso.

Aos colegas também alunos desta Pós-Graduação, pela convivência enriquecedora, especialmente aos colegas Luiz Albino e Rafael Moraes, pela parceria em trabalhos, e Daiane Rodrigues e Soraida Aguilar, flores neste caminho.

A todos os meus amigos pessoais que me incentivavam, torciam, compreendiam minhas ausências e estiveram sempre do meu lado, especialmente ao meu amigo Tinus van Vugt pela sua presença nesta fase da minha vida, apoio, carinho e dedicação.

À UFF e todos os colegas professores do Departamento de Estatística pela licença concedida para eu me dedicar ao doutorado.

Aos meus chefes superiores no CEDERJ e UFF, Masako Masuda, Ana Maria, Celso Costa e Regina Moreth por todo apoio e compreensão.

À PUC-Rio pelo acolhimento, seus funcionários e recursos físicos.

Aos membros da Banca Examinadora pelas importantes contribuições.

A todos os autores referenciados, que enriquecem meu trabalho com suas pesquisas.

À cidade maravilhosa do Rio de Janeiro e seu Samba, paixões da minha vida, que neutralizam todo stress e me dão tanta alegria.

Ao Povo Brasileiro, financiador direto desta conquista. 


\section{Resumo}

Cassiano, Keila Mara; Souza, Reinaldo Castro (Orientador). Análise de Séries Temporais Usando Análise Espectral Singular (SSA) e Clusterização de Suas Componentes Baseada em Densidade. Rio de Janeiro, 2014, 172p. Tese de Doutorado - Departamento de Engenharia Elétrica, Pontifícia Universidade Católica do Rio de Janeiro.

Esta tese propõe a utilização do DBSCAN (Density Based Spatial Clustering of Applications with Noise) para separar os componentes de ruído na fase de agrupamento das autotriplas da Análise Singular Espectral (SSA) de Séries Temporais. O DBSCAN é um método moderno de clusterização (revisto em 2013) e especialista em identificar ruído através de regiões de menor densidade. O método de agrupamento hierárquico até então é a última inovação na separação de ruído na abordagem SSA, implementado no pacote R- SSA. No entanto, o método de agrupamento hierárquico é muito sensível a ruído, não é capaz de separá-lo corretamente, não deve ser usado em conjuntos com diferentes densidades e não funciona bem no agrupamento de séries temporais de diferentes tendências, ao contrário dos métodos de aglomeração à base de densidade que são eficazes para separar o ruído a partir dos dados e dedicados para trabalhar bem em dados a partir de diferentes densidades. Este trabalho mostra uma melhor eficiência de DBSCAN sobre os outros métodos já utilizados nesta etapa do SSA, garantindo considerável redução de ruídos e proporcionando melhores previsões. O resultado é apoiado por avaliações experimentais realizadas para séries simuladas de modelos estacionários e não estacionários. A combinação de metodologias proposta também foi aplicada com sucesso na previsão de uma série real de velocidade do vento.

\section{Palavras-chave}

Análise Singular Espectral; Clusterização Baseada em Densidade; DBSCAN; Mineração de Dados; Previsão; Séries Temporais; Previsão SSA; Modelos ARIMA; Energia Eólica. 


\section{Abstract}

Cassiano, Keila Mara; Souza, Reinaldo Castro (Advisor). Time Series Analysis Using Singular Spectrum Analysis (SSA) and Based Density Clustering of The Components. Rio de Janeiro, 2014, 172p. Doctorate Thesis - Departamento de Engenharia Elétrica, Pontifícia Universidade Católica do Rio de Janeiro.

This thesis proposes using DBSCAN (Density Based Spatial Clustering of Applications with Noise) to separate the noise components of eigentriples in the grouping stage of the Singular Spectrum Analysis (SSA) of Time Series. The DBSCAN is a modern (revised in 2013) and expert method at identify noise through regions of lower density. The hierarchical clustering method was the last innovation in noise separation in SSA approach, implemented on package R-SSA. However, is repeated in the literature that the hierarquical clustering method is very sensitive to noise, is unable to separate it correctly, and should not be used in clusters with varying densities and neither works well in clustering time series of different trends. Unlike, the methods of density based clustering are effective in separating the noise from the data and dedicated to work well on data from different densities This work shows better efficiency of DBSCAN over the others methods already used in this stage of SSA, because it allows considerable reduction of noise and provides better forecasting. The result is supported by experimental evaluations realized for simulated stationary and non-stationary series. The proposed combination of methodologies also was applied successfully to forecasting real series of wind's speed.

\section{Keywords}

Singular Spectrum Analysis; Density Based Clustering; DBSCAN; Data Mining; Forecast; Time Series; SSA Forecast; ARIMA Models; Wind Energy. 


\section{Sumário}

1. Introdução 18

1.1. Motivação 19

1.2. Objetivo do Trabalho 21

1.3. Relevância do Tema 21

1.4. Organização da Tese 22

2. Séries Temporais 23

2.1. Definição 23

2.2. O Operador de Diferenças 26

2.3. Processo Ruído Branco 27

2.4. Processo Passeio Aleatório 27

2.5. Modelos ARIMA 28

2.6. Medidas de Qualidade de Ajuste 32

3. Singular Spectrum Analysis (SSA) 34

3.1. Decomposição SSA 36

3.1.1. Incorporação 36

3.1.2. Singular Value Decomposition (SVD) 37

3.2. Reconstrução SSA 41

3.2.1. Agrupamento 41

3.2.2. Média Diagonal 42

3.3. Métodos de Agrupamento 45

3.3.1. Agrupamento por Análise de Componentes Principais 46

3.3.2. Agrupamento por Análise Gráfica dos Autoverores

Singulares 46

3.3.2.1. Análise do Periodograma 47

3.3.3. Agrupamento por Clusterização 48

$\begin{array}{lll}\text { 3.4. Separabilidade } & 48\end{array}$ 
3.5. Escolha do Parâmetro $L$ de Defasagem 50

3.6. Previsão SSA 53

3.7. SSA na Análise de Séries Temporais 55

4. Clusterização de Dados 59

4.1. Aplicações 60

4.2. Limitação 62

4.3. Medidas de Similaridade 62

4.4. Métodos de Clusterização 67

4.4.1. Métodos Hierárquicos 69

4.4.2. Métodos Particionais 70

4.4.3. Métodos Baseados em Densidade 72

4.4.4. Métodos Baseados em Grade 73

4.4.5. Métodos Baseados em Modelos 74

4.4.6. Métodos Baseados em Redes Neurais Artificiais 74

4.4.7. Métodos Baseados em Lógica Fuzzy 75

4.4.8. Métodos Baseados em Kernel 76

4.4.9. Métodos Baseados em Grafos 76

4.4.10. Métodos Baseados em Computação Evolucionária 77

4.4.11. Histórico dos Métodos de Clusterização 78

5. Método DBSCAN 97

5.1. Algoritmo DBSCAN 104

5.2. DBSCAN Revisado 106

5.3. Avaliação de Performance e Aplicações do DBSCAN 110

6. Metodologia 114

6.1. Processos Geradores de Dados Simulados 115

6.2. Ferramentas Computacionais 116

7. Resultados das Avaliações Dados Simulados 117

8. Aplicação da Metodologia à Série de Velocidade do Vento 120 
8.1. Modelagem e Previsão Tradicional (sem SSA) 122

8.2. Modelagem e Previsão usando SSA 131

8.2.1. Identificação do Ruído por Verificação Visual dos Vetores Singulares

8.2.2. Identificação do Ruído por Análise de Componentes

Principais

8.2.3. Identificação do Ruído pelo Método de Clusterização

Hierárquica

8.2.4. Identificação do Ruído pelo Método de Clusterização DBSCAN

8.3. Previsão Mista: Filtro SSA + Previsão Clássica

8.3.1. Modelagem para a série aproximada obtida por verificação visual dos autovetores

8.3.2. Modelagem para a série aproximada obtida por ACP

8.3.3. Modelagem para a série aproximada obtida por Clusterização Hierárquica

8.3.4. Modelagem para a série aproximada obtida por Clusterização DBSCAN

8.4. Previsão SSA: Filtro SSA + Previsão SSA

8.5. Comparação dos Resultados da Previsão Clássica, Previsão Mista e Previsão SSA

9. Conclusões

10. Referências Bibliográficas 


\section{Lista de Figuras}

Figura 3.1. Estimação de L para filtragem SSA

Figura 4.1. Conjuntos com clusters globulares, não globulares e com ruídos

Figura 5.1. $\varepsilon$-vizinhança de q e $\varepsilon$-vizinhança de $p \quad 98$

Figura 5.2. Alcance direto por densidade no método DBSCAN 99

Figura 5.3. Alcance por densidade no método DBSCAN 100

Figura 5.4: Conexão por densidade no método DBSCAN 100

Figura 5.5. Pseudo Código do Algoritmo DBSCAN - Subrotina Principal

105

Figura 5.6. Pseudo Código do Algoritmo DBSCAN - Subrotina de Expansão dos Clusters

Figura 5.7. Pseudo Código do Algoritmo DBSCAN- Revisado Subrotina Principal

Figura 5.8. Pseudo Código do Algoritmo DBSCAN- Revisado Subrotina de Expansão dos Clusters

Figura 5.9. Desempenho de Diferentes Métodos de Clusterização para Dados Espaciais

Figura 7.1. Amostras de cada um dos processos estacionários simulados

Figura 7.2: Amostras de cada um dos processos não estacionários simulados

Figura 8.1. Curva de Potência Elétrica Produzida em função da velocidade do vento

Figura 8.2. Médias mensais de velocidade do vento na Estação

Petrolina - PE

Figura 8.3. Box Plot e Histograma da Distribuição de velocidade do vento na Estação Petrolina - PE

Figura 8.4. Boxplots das médias de velocidade de vento na Estação de Petrolina

Figura 8.5. Função de Autocorrelação da série de Velocidade do 
Figura 8.6. Função de Autocorrelação Parcial da série de

Velocidade do Vento de Petrolina

Figura 8.7. Função da Autocorrelação da série $\nabla_{12}^{1} Y_{\mathrm{t}}$. 127

Figura 8.8. Função da Autocorrelação Parcial da série $\nabla_{12}^{1} Y_{\mathrm{t}}$. 128

Figura 8.9. Estimação do modelo SARIMA $(1,0,0) \times(1,0,1)_{12}$ para $\mathrm{Y}_{\mathrm{t}}$ pelo $\mathrm{FPW}$

128

Figura 8.10. Estimação do modelo SARIMA $(1,0,0) \times(1,0,1)_{12}$ para $\mathrm{Y}_{\mathrm{t}}$ pelo $\mathrm{FPW}$.

Figura 8.11. Série dos resíduos do modelo SARIMA $(1,0,0) \times$ $(1,0,1)_{12}$

Figura 8.12. Autocorrelação dos resíduos do modelo

130

Figura 8.13. Logaritmo dos autovalores da matriz $X X^{T}$

Figura 8.14. Os seis primeiros vetores singulares em ordem de significância

132

Figura 8.15. Alguns diagramas de dispersão

Figura 8.16. Periodograma dos vetores singulares 2 e 3 .

134

Figura 8.17. Componentes da metodologia SSA identificados por Verificação Visual do Comportamento dos Autovetores.

Figura 8.18. Série de Sinal Identificado por SVD

Figura 8.19. Série de Ruído identificado por SVD.

Figura 8.20. Série de Sinal Determinado por Clusterização

Hierárquica.

138

Figura 8.21. Série de Ruído Determinada por Clusterização

Hierárquica.

Figura 8.22: Dist4 dos dados de Petrolina para a estimação de $\varepsilon$ pro algoritmo DBSCAN.

Figura 8.23. Série de Sinal Identificada por DBSCAN.

Figura 8.24. Série de Ruído Identificada por DBSCAN.

Figura 8.25: Resíduos do Modelo ARIMA para séries filtradas em SSA através da Verificação Visual dos Autovetores, calculados em relação à série original 
Figura 8.26 : Resíduos do Modelo ARIMA para séries filtradas em SSA através da Análise de Componentes Principais, calculado em relação à série original

Figura 8.27: Resíduos do Modelo ARIMA para séries filtradas em SSA através da Clusterização Hierárquica, calculados em relação 144 à série original

Figura 8.28: Resíduos do Modelo ARIMA para séries filtradas em SSA através da DBSCAN, calculados em relação à série original Figura 8.29: Resíduos da Previsão SSA para séries filtradas através da Verificação Visual do Comportamento dos Autovetores Figura 8.30: Resíduos da Previsão SSA para séries filtradas através da Análise de Componentes Principais.

Figura 8.31 : Resíduos da Previsão SSA para séries filtradas através da Clusterização Hierárquica.

Figura 8.32 : Resíduos da Previsão SSA para séries filtradas através do DBSCAN 


\section{Lista de Quadros e Tabelas}

Quadro 4.1: Evolução dos Métodos de Clusterização

89

Tabela 7.1: Média do MAPE (in sample) da previsão de séries de Modelos Simulados, em cada uma das abordagens propostas, a partir das 100 replicações

Tabela 7.2: Média do RMSE (in sample) da previsão de séries de Modelos Simulados em cada uma das abordagens propostas, a partir das 100 replicações

Tabela 8.1. Principais Estatísticas da Série Original de Petrolina

Tabela 8.2: Principais estatísticas, por mês, das medidas de velocidade do vento em Petrolina/BA.

Tabela 8.3. Definições das componentes pelo método da Verificação Visual.

Tabela 8.4. Teste BDS aplicado a série de ruídos identificados por Verificação Visual.

Tabela 8.5. Correlação ponderada entre as componentes identificadas por Verificação Visual.

Tabela 8.6. Teste BDS aplicado a série de ruídos identificados por SVD.

Tabela 8.7. Teste BDS aplicado à série de ruídos identificados pela Clusterização Hierárquica.

Tabela 8.8. Definições das componentes pelo método DBSCAN.

Tabela 8.9. Teste BDS aplicado a série de ruídos identificados por DBSCAN

Tabela 8.10: Medidas de qualidade de ajuste das previsões in sample da série de Petrolina por todos os métodos experimentados.

Tabela 8.11: Medidas de qualidade de ajuste das previsões 24 passos a frente out of sample da série de Petrolina por todos os métodos experimentados. 
Tabela 8.12. Previsões passos a frente pelo método SSA com DBSCAN e Previsão SSA para a série de velocidade do vento da estação de Petrolina 
Não entendo.

Isso é tão vasto que ultrapassa qualquer entender.

Entender é sempre limitado, mas não entender pode não ter fronteiras.

Sinto que sou muito mais completa quando não entendo.

O bom é ser inteligente e não entender.

É uma benção estranha, como ter loucura sem ser doida.

É um desinteresse manso, é uma doçura de burrice.

Só que de vez em quando vem a inquietação, quero entender um pouco..." 